\title{
A SILICON MEMS DISK RESONATOR OSCILLATOR DEMONSTRATING 36 PPT FREQUENCY STABILITY
}

\author{
Madan Parajuli, Guillermo Sobreviela, Hemin Zhang, and Ashwin A. Seshia \\ Nanoscience Centre, Department of Engineering, University of Cambridge, Cambridge, UK
}

\begin{abstract}
This paper reports experimental results demonstrating excellent short-term frequency stability of $45.6 \mu \mathrm{Hz}$ (36 ppt (a $0.4 \mathrm{~s}$ integration time) for a bulk acoustic wave (BAW) silicon disk resonator oscillator. The $n=4$ radial mode of a BAW disk resonator demonstrates an extremely highquality factor of $1.8 * 10^{6}$ at $1.25 \mathrm{MHz}$. The disk is designed with anchors aligned with nodal locations to minimize anchor damping. The results on the measured short-term frequency stability reported here benchmark favourably relative to the state-of-the-art.
\end{abstract}

\section{KEYWORDS}

MEMS oscillators, bulk mode resonators, frequency stability.

\section{INTRODUCTION}

MEMS resonators and oscillators have been the focus of much interest in recent years for their usage in a wide range of diverse applications such as timing and frequency control [1], high-performance inertial sensors [2], chemical sensing [3] and biological sensing [4]. MEMS technologies demonstrate several advantageous features such as miniaturization, batch manufacturability, and close integration with CMOS electronics [1], [5], [6] making MEMS oscillators attractive alternatives to well established quartz-based oscillators for timing and frequency control applications. Additionally, they demonstrate excellent short-term and long-term frequency stability, with temperature sensitivity reduced through the integration of passive [7] and active [8] compensation schemes. The reported performance of MEMS oscillators have improved significantly in recent years, displaying sub-ppb short-term and long-term frequency stabilities

A wide variety of structural topologies of MEMS oscillators has been investigated. Specifically, attention has focussed on flexural mode [9] and bulk acoustic mode [10] devices. Compared to flexural mode resonators, bulk mode MEMS resonators display good scaling characteristics and have better intrinsic immunity to shock and vibration. The frequency stability of the resonator improves with a high quality factor making bulk mode resonators popular in timing and frequency control applications.

In this work, excellent frequency stability is demonstrated using a higher order bulk mode (radial $n=4$ ) silicon disk resonator competing with the best results in the current state-of-the-art. The short term frequency stability demonstrated in this paper is 1.4 times better than the frequency stability enhanced through degenerate mode locking in a silicon MEMS oscillator reported in a previous study [11]. Table 1 provides a state-of-the-art benchmark comparison of the short-term frequency stability for previously reported MEMS oscillators and commercial crystal oscillators and compares these values to the short-term stability reported in this work.

Table 1: Comparison of the short-term frequency stability for selected silicon and commercial crystal oscillators.

\begin{tabular}{|c|c|c|}
\hline Reference & Frequency & Short-term stability \\
\hline$[11]$ & $976 \mathrm{kHz}$ & $0.051 \mathrm{ppb}$ \\
\hline$[12]$ & $1.18 \mathrm{MHz}$ & $0.34 \mathrm{ppb}$ \\
\hline$[13]$ & 5 to $20 \mathrm{MHz}$ & $0.4 \mathrm{ppb}$ \\
\hline$[14]$ & $2.18 \mathrm{MHz}$ & $0.6 \mathrm{ppb}$ \\
\hline$[15]$ & $139 \mathrm{kHz}$ & $6.7 \mathrm{ppb}$ \\
\hline$[16]$ & $1.62 \mathrm{MHz}$ & $10 \mathrm{ppb}$ \\
\hline$[17]$ & $10.09 \mathrm{MHz}$ & $18 \mathrm{ppb}$ \\
\hline This work & $\mathbf{1 . 2 5} \mathbf{M H z}$ & $\mathbf{0 . 0 3 6} \mathbf{~ p p b}$ \\
\hline
\end{tabular}

\section{DEVICE}

The device-under-test in this study is a vacuum packaged micromachined disk resonator fabricated in a siliconon-insulator (SOI) MEMS process employing wafer-level vacuum encapsulation [2]. Figure 1 shows an optical micrograph of the device. The disk has a radius of $3 \mathrm{~mm}$ and a thickness of $40 \mu \mathrm{m}$. The disk is surrounded by 12 identical electrodes and employs edge anchoring. The characterisation of the resonant modes for this device has revealed several modes with quality factors exceeding a million [12], [18] and a Q-f metric exceeding $10^{13}$. The frequency response showing all in-plane modes between $500 \mathrm{kHz}$ to $2 \mathrm{MHz}$ is presented in Fig. 2. The mode of interest is the radial $n=4$ mode illustrated in Fig. 3 .

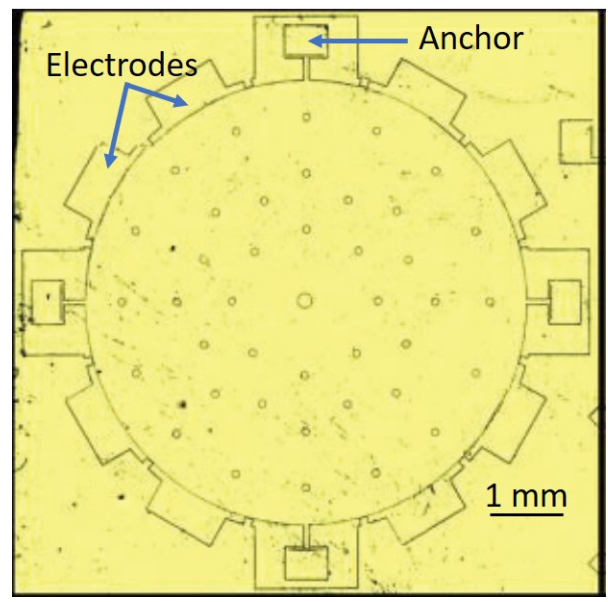

Figure 1: Optical micrograph of the MEMS device.

\section{EXPERIMENTAL SETUP}

The experimental setup shown in Fig. 4 is used to drive the resonator in closed loop. The resonator is placed in a temperature-controlled chamber with a nominal temperature stability of $\pm 1 \mathrm{mK}$. The motional current is amplified 


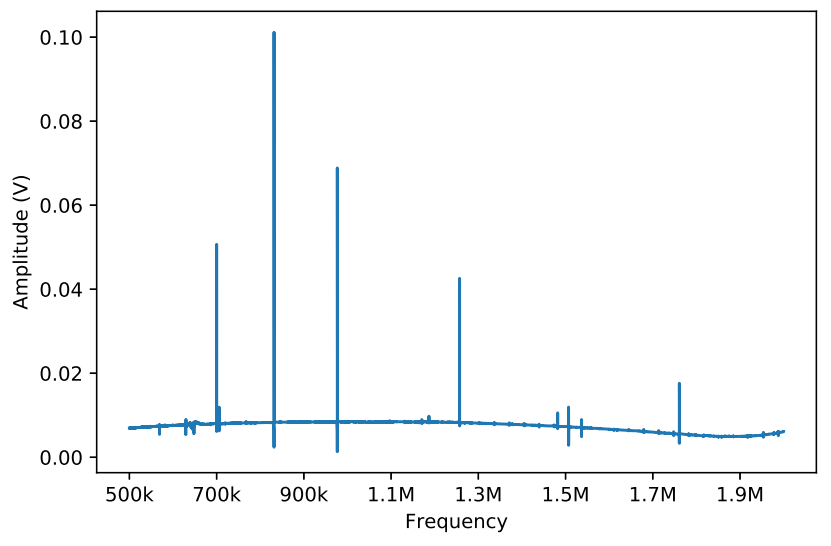

Figure 2: Measured frequency response of the MEMS resonator showing all in-plane modes between $500 \mathrm{kHz}$ to $2 \mathrm{MHz}$.

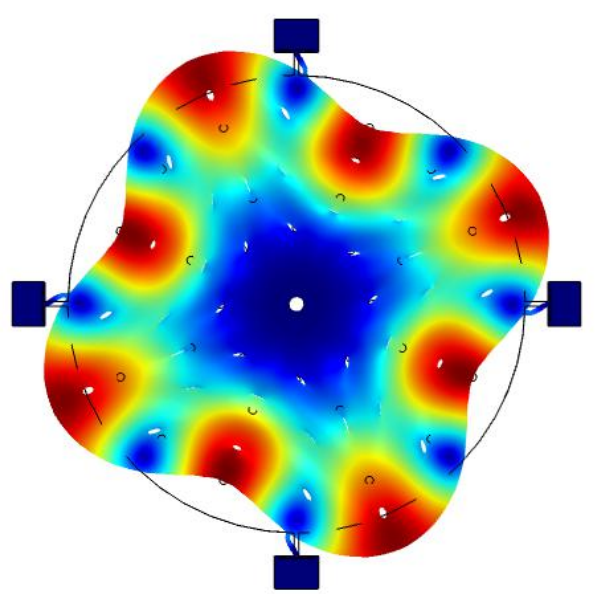

Figure 3: COMSOL simulation results showing radial, $n=4$ mode. Colours indicate absolute displacement: red max (anti-node), dark blue zero (node)

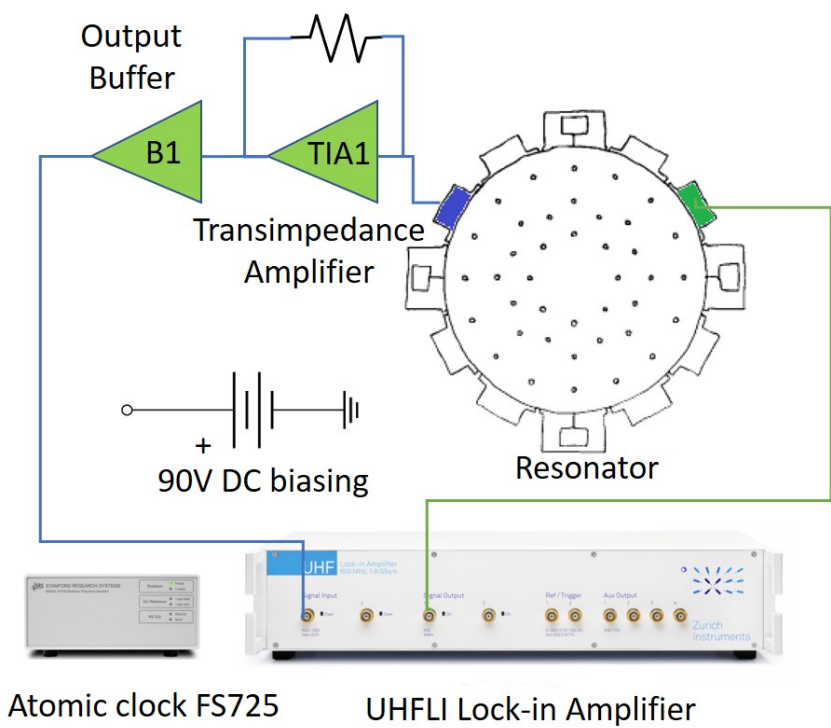

Figure 4: Schematic of experimental setup. by a low-noise transimpedance amplifier and the buffered output is then input to a Zurich Instruments UHFLI Lockin Amplifier with an atomic clock FS725 as an external clock reference. The resonator is biased with a DC voltage of $90 \mathrm{~V}$ and a $1.5 \mathrm{Vrms}$ actuation signal is employed. The resonant frequency and phase obtained from openloop characterisation were used to implement the feedback configuration using the PLL function of the UHFLI Lockin Amplifier.

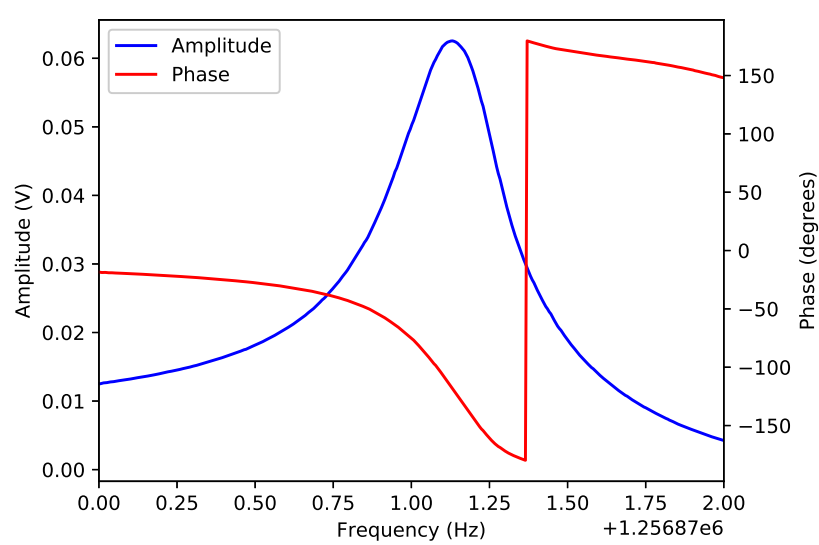

Figure 5: Measured frequency response of the MEMS resonator.

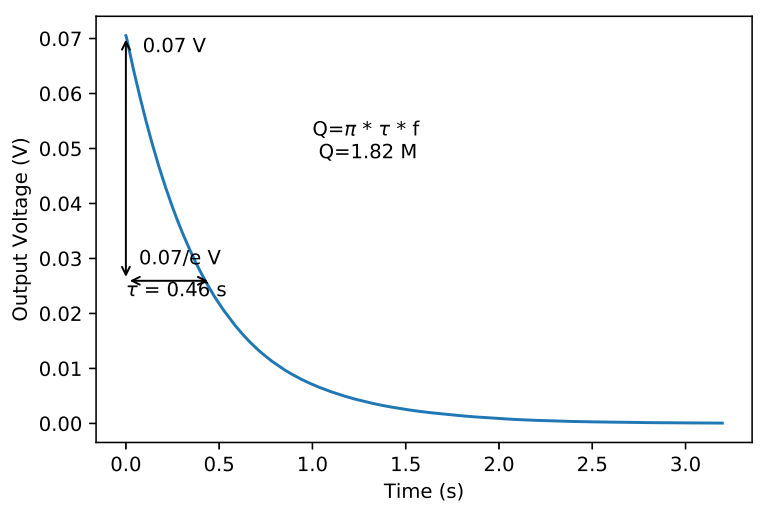

Figure 6: Measured ring down response of the MEMS resonator.

\section{RESULTS}

The measured open-loop frequency response of the resonator is presented in Fig. 5. A ring-down experiment is conducted and the resulting time domain response of the output is presented in Fig. 6. The ring-down time $(\tau)$ is $0.46 \mathrm{~s}$ corresponds to a quality factor of $1.82 * 10^{6}$. A key figure of merit for resonators is the f.Q product. For the device-under-test, this value is $2.2 * 10^{12}$. 


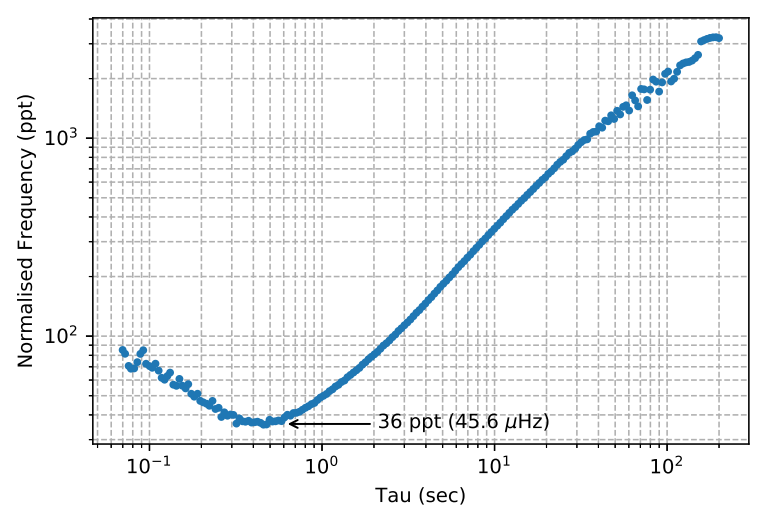

Figure 7: Measured Allan deviation of the output frequency of the MEMS oscillator

In order to determine the frequency stability of the oscillator, the UHFLI is adapted as a digital PLL and the output frequency is logged. The Allan deviation plot in Fig. 7 shows a frequency stability of $45.6 \mu \mathrm{Hz}$ (normalized frequency stability of $36 \mathrm{ppt}$ ) at an integration time of 0.4 s.

To evaluate the consistency of the short-term stability, a 2 hours and 10 minutes long measurement is conducted: the recorded frequency data is divided into 13 samples and the Allan deviation for each sample is plotted in Fig. 8. The results are very consistent across the entire dataset, with a recorded minimum Allan deviation varying between $36 \mathrm{ppt}$ to $42 \mathrm{ppt}$

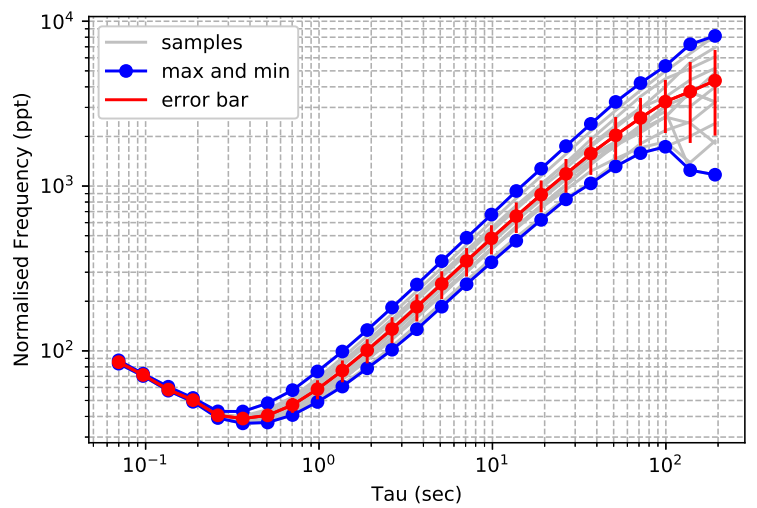

Figure 8: Allan deviation measured for 13 independent runs, each obtained for 130 minutes sampling.

A longer-term measurement (9 hours and 28 minutes in duration) is conducted to evaluate the frequency stability of the MEMS oscillator for longer integration times. Figure 9 shows that the frequency stability for an integration time of 2 hours is $0.3 \mathrm{ppm}$. The drift in the output frequency is dominated by temperature variations as the resonator in this study is not temperature compensated

The frequency stability of the commercial waveform generator Agilent 33250A and Zurich Instruments UHFLI are measured using a frequency counter (Agilent 53230A). The atomic clock FS725 is used as an external clock reference to the frequency counter. The Allan deviation measurements of $33250 \mathrm{~A}$ and UHFLI are compared to

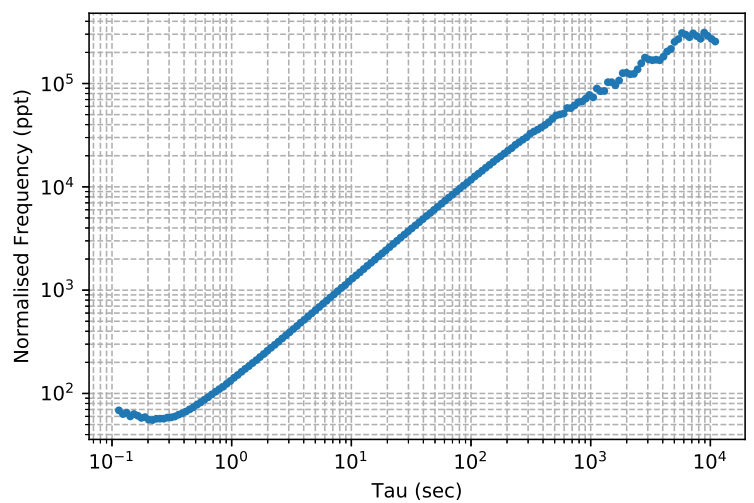

Figure 9: Allan deviation measured over a longer duration showing the impact of long-term drift on the measurement.

the stability results of the device used in the experiments described above. Figure 10 shows that the oscillator displays better short-term frequency stability relative to the output derived from two existing instruments at the same frequency.

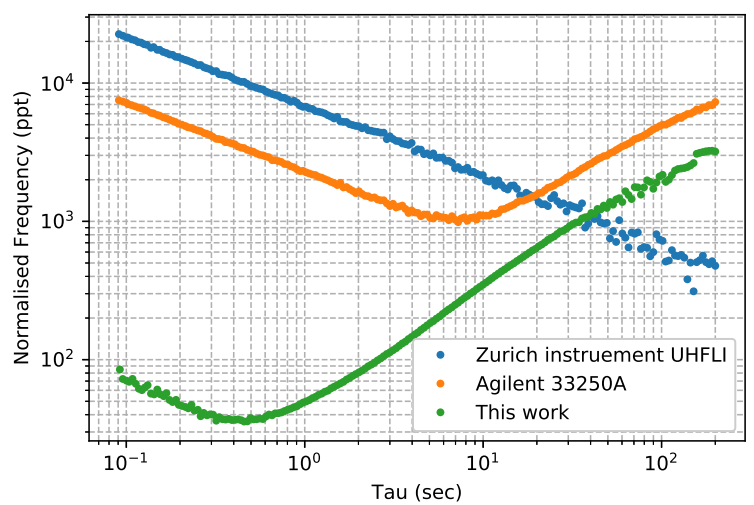

Figure 10: Allan deviation measurements for the Zurich Instruments UHFLI, commercial waveform generator Agilent $33250 \mathrm{~A}$ and locked MEMS oscillator (this work).

\section{SUMMARY}

The 1.2 MHz bulk mode MEMS resonator, fabricated in a SOI MEMS process, presented in this paper displays a high quality factor of 1.8 million. The MEMS resonator was embedded in a feedback loop to realize an oscillator with a short-term output frequency stability of $36 \mathrm{ppt}$, a result that is better than other highly engineered quartz crystal oscillators. Further improvement in the long-term frequency stability can be obtained using temperature compensation. Such bulk-mode MEMS oscillators combined with active and passive temperature compensation schemes could enable applications to stable time and frequency references, as well as resonant sensors such as gyroscopes.

\section{ACKNOWLEDGMENTS}

This work was supported by funding from Engineering and Physical Research Council UK, Silicon Microgravity and Innovate UK. 


\section{REFERENCES}

[1] C. T-C. Nguyen. Mems technology for timing and frequency control. IEEE Transactions on Ultrasonics, Ferroelectrics, and Frequency Control, 54(2):251-270, 2007.

[2] A. Mustafazade, M. Pandit, C. Zhao, G. Sobreviela, Z. Du, P. Steinmann, X. Zou, R. T. Howe, and A. A. Seshia. A vibrating beam mems accelerometer for gravity and seismic measurements. Scientific reports, 10(1):1-8, 2020.

[3] M. Rinaldi, B. Duick, C. Zuniga, C. Zuo, and G. Piazza. Ss-dna functionalized ultra-thin-film aln contourmode resonators with self-sustained oscillator for volatile organic chemical detection. In 2010 IEEE 23rd International Conference on Micro Electro Mechanical Systems (MEMS), pages 132-135, 2010.

[4] E. Mehdizadeh, J. C. Chapin, J. M. Gonzales, A. Rahafrooz, R. Abdolvand, B. W. Purse, and S. Pourkamali. Microelectromechanical disk resonators for direct detection of liquid-phase analytes. Sensors and Actuators A: Physical, 216:136-141, 2014.

[5] J. T. M. Van Beek and R. Puers. A review of mems oscillators for frequency reference and timing applications. Journal of Micromechanics and Microengineering, 22(1):013001, 2011.

[6] W-T. Hsu. Recent progress in silicon mems oscillators. Technical report, Discera Inc Ann Arbor MI, 2008.

[7] A. K. Samarao and F. Ayazi. Temperature compensation of silicon resonators via degenerate doping. IEEE Transactions on Electron Devices, 59(1):87-93, 2012.

[8] M. Heidarpour Roshan, S. Zaliasl, K. Joo, K. Souri, R. Palwai, L. W. Chen, A. Singh, S. Pamarti, N. J. Miller, J. C. Doll, C. Arft, S. Tabatabaei, C. Sechen, A. Partridge, and V. Menon. A mems-assisted temperature sensor with $20-\mu \mathrm{K}$ resolution, conversion rate of $200 \mathrm{~s} / \mathrm{s}$, and fom of 0.04 pjk2. IEEE Journal of Solid-State Circuits, 52(1):185-197, 2017.

[9] S. Pourkamali, A. Hashimura, R. Abdolvand, G. K. Ho, A. Erbil, and F. Ayazi. High-q single crystal silicon harpss capacitive beam resonators with selfaligned sub-100-nm transduction gaps. Journal of Microelectromechanical Systems, 12(4):487-496, 2003.

[10] X. Zou, C. Zhao, and A. A. Seshia. Edgeanchored mode-matched micromachined gyroscopic disk resonator. In 2017 19th International Conference on Solid-State Sensors, Actuators and Microsystems (TRANSDUCERS), pages 2183-2186, 2017.

[11] M. Parajuli, G. Sobreviela, H. Zhang, and A. A. Seshia. Enhancement of frequency stability in injection locked bulk mode mems oscillators. In 2021 IEEE 34th International Conference on Micro Electro Mechanical Systems (MEMS), pages 941-944, 2021.

[12] G. Sobreviela, X. Zou, C. Zhao, M. Pandit, and A. A. Seshia. An ultra-high-quality factor silicon disk resonator. In 2019 20th International Conference on Solid-State Sensors, Actuators and Microsystems Eurosensors XXXIII (TRANSDUCERS EUROSENSORS XXXIII), pages 527-530, 2019.
[13] Vectron. "high stability coefficient oscillator $\mathrm{i}^{2} \mathrm{c}$ interface oven controlled crystal oscillator (md-173)", sep. 15, 2020. [online]. available: https://www.vectron.com/products/ocxo/md-173.pdf.

[14] J. E-Y. Lee, B. Bahreyni, Y. Zhu, and A. A. Seshia. A single-crystal-silicon bulk-acoustic-mode microresonator oscillator. IEEE Electron Device Letters, 29(7):701-703, 2008.

[15] X. Zou, P. Thiruvenkatanathan, and A. A. Seshia. A high-resolution micro-electro-mechanical resonant tilt sensor. Sensors and Actuators A: Physical, 220:168177, 2014.

[16] E. J. Ng, H. K. Lee, C. H. Ahn, R. Melamud, and T. W. Kenny. Stability of silicon microelectromechanical systems resonant thermometers. IEEE Sensors Journal, 13(3):987-993, 2013.

[17] Y. Yang, E. J. Ng, Y. Chen, I. B. Flader, and T. W. Kenny. A unified epi-seal process for fabrication of high-stability microelectromechanical devices. Journal of Microelectromechanical Systems, 25(3):489-497, 2016.

[18] M. Parajuli, G. Sobreviela, and A. A. Seshia. Electrostatic frequency tuning of bulk acoustic wave disk gyroscopes. In 2020 Joint Conference of the IEEE International Frequency Control Symposium and International Symposium on Applications of Ferroelectrics (IFCS-ISAF), pages 1-4, 2020.

\section{CONTACT}

Madan Parajuli, mp912@cam.ac.uk 